\title{
A Study of DiffServ Based QoS Issues in Next Generation Mobile Networks
}

\section{Thimma V.J. Ganesh Babu}

Ryerson University

\author{
A. Anpalagan \\ Ryerson University \\ Jeremiah F. Hayes
}

Concordia University

Please Cite:

Babu, T. V. J. G., Anpalagan, A., \& Hayes, J. F. (2004). A study of DiffServ based QoS issues in next generation mobile networks. Canadian Conference on Electrical and Computer Engineering, 4, 2359-2362.

$\underline{\text { doi:10.1109/CCECE.2004.1347720 }}$

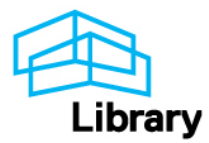




\section{A Study of DiffServ based QoS Issues in Next Generation Mobile Networks}

\author{
Thimma V.J. Ganesh Babu, A. Anpalagan \\ Ryerson University \\ ELCE Department \\ 350 Victoria Street, Toronto \\ Ontario, M5B 2K3
}

\author{
Jeremiah F. Hayes \\ Concordia University \\ ECE Department \\ 1455 De Maisonneuve Blvd. \\ Montreal, Quebec, H3G 1M8
}

\begin{abstract}
To provide data rates of the order of hundreds of Mbps and multimedia services, standardization efforts for next generation(4G) systems are focusing on target technologies and seamless connectivity through various types of networks including wireline networks and WLANs. Different types of multiple access techniques such as the ones based on multi-carrier CDMA, and OFDM(Orthogonal Frequency Division Multiplexing) have been proposed. There is a need for functional integration of the multiple networks and with the evolution of IPv6 and QoS support for IP networks, an IP based interconnectivity is best suited. A QoS aware adaptive radio resource management techniques based on multi-code multicarrier CDMA is discussed. We develop a novel radio access method and develop algorithms for allocating and controlling radio network resources so that system performance can be maximized and guaranteed QoS of multimedia services can be provided within the DiffServ environment.
\end{abstract}

Keywords - Multicode multicarrier CDMA, Diffserv, 4G, QoS, IPv6, power control, rate control

\section{INTRODUCTION}

To provide data rates of the order of hundreds of Mbps and multimedia services, standardization efforts for next generation(4G) systems are focusing on target technologies and seamless connectivity through various types of networks including wireline networks and WLANs. Different types of multiple access techniques such as the ones based on multi-code multicarrier CDMA, and OFDM(Orthogonal Frequency Division Multiplexing) have been proposed. With technologies such as UltraWide Band(UWB) modulation technique and Multiple Input Multiple Output(MIMO) antenna architectures, expected high data rates of $4 \mathrm{G}$ systems can be provided. There is a need for functional integration of the multiple networks and with the evolution of IPv6 and QoS support for IP networks, an IP based interconnectivity is best suited. We develop QoS aware radio resource management techniques based on different types of radio access technologies such as multi-code multi-carrier CDMA, OFDM, etc. We propose novel radio access methods and develop algorithms for allocating and controlling radio network resources so that system performance can be maximized and guaranteed QoS of multimedia services can be provided within the DiffServ environment. We study the effect of QoS aware radio resource management algorithms on both time critical and non time critical applications typically in wide use in today's networks and suggest required modification on protocol functionalities in order to provide seamless connectivity in a mobile environment using various types of interconnected networks.

Radio resource control in order to provide QoS, has been part of $2 \mathrm{G}$ and $3 \mathrm{G}$ specifications and is referred as the Radio Resources Management(RRM) which provides QoS support to network layer through data link and eventually through physical layer[1]. For the next generation wireless networks, an Adaptive Resource Management(ARM) and control is needed in order to support multiple layer capabilities. By adjusting the parameters and functions as needed in order to optimize features such as QoS, throughput, power utilization, etc., ARM stands out as a multi-layer functionality. For example throughput requirement can arise from transport layer(typically from TCP connections). Typical adaptability can be provided through multiple modulation, coding 
and multiple access schemes at the physical layer. This in turn can result in adaptively setting the weights of WFQ scheduler, when the traffic pattern changes or when the higher layers renegotiate their QoS. In this paper, we consider at the physical layer multi-code multi-carrier CDMA as the radio access scheme used by mobiles. In fact, from the point of view of BER performance it is shown that multi-code MCCDMA, outperforms OFDM for the same data rate and bandwidth with frequency selective fading channel[2]. Our focus here is to provide a methodology for supporting rate control and power control in order to support the required QoS for Diffserv classes.

In Section 2, we discuss the typical parameters and options available to deal with mutli-code MC-CDMA. We assume Rayleigh fading channel. In Section 3, we discuss how the Diffserv classes requirements in terms of delay, loss, and throughput can be mapped on to the parameters at the physical layer. Finally in Section 4 , we provide our conclusions.

\section{MULTI-CODE MC CDMA}

There are two ways by which multi-code MCCDMA can be implemented. The original data stream can be spread by using a spreading code and then can be modulated by a different subcarrier with each chip. In the other way, original data stream is first converted from serial to parallel data stream, and then spread by a given spreading code and modulated by a different subcarrier with each of parallel stream similar to a normal DS-CDMA scheme[3]. In both cases, apart from spreading with single code, multiple codes can be used with different bits on the same subcarrier. The performance analysis of MC CDMA has been performed in [4] for multipath fading channel. As we can see from this analysis(Equation 38), the probability of bit error is complex function of power of the bit, and interference which are due to same and other users of the same carrier, and other carrier interference. Once again given a set of carriers, bit error can be controlled by controlling the power with which a user can transmit.
We impose a power control mechanism in order to control packet losses. The optimal power control will be the one which meets the given target SINR(signal to interference and noise ratio) at the same time minimizes the total transmitted power. For the simple case of DSCDMA an explicit expression has been shown in [5]. For the case of multi-code MC-CDMA this will be a formidable task. But, it can be easily performed in simulation. The rate control can be achieved by controlling the number of codes that can be assigned for the same user within the subcarriers. Note that we can have different number of codes in different subcarriers which will result in lower interference but would yield higher rate for the user, within the imposed maximum power constraint. The bits belonging to each packet will be requesting a particular SINR to be supported. The system depending on the conditions should provide this minimum SINR requested. If all the SINR requests from all mobile stations cannot be satisfied, then some of the mobile stations can be shut off. It means they will not able to transmit until the base station controller enables transmission when the required minimum SINR can be supported[5].

\section{DIFFSERV QOS}

Ipv6 Diffserv architecture, proposes, different types of per hop behaviour classes. The widely proposed classes can be classified as according to which per hop behaviour class it belongs to. There are Expedited forwarding PHB class, Assured forwarding PHB classes(there four different classes and within each class there are three levels of loss) and Best Effort PHB class(this PHB is defined in order to be compatible to legacy classes of Ipv4). In Ipv6 packet, the DSCP(Differentiated Services Code Point) identifies the type of class a packet belongs to. An IP packet entering into the Diffserv domain, will first be mapped into appropriate class by choosing appropriate DSCP and then it will be subjected to Traffic Conditioning functions, at the DS ingress node. EF PHB class of packets can be mapped to real time traffic which has stringent delay 
requirements. AF PHB classes of traffic can be used for TCP types of traffic, for example, http traffic which has the strict throughput requirement. Also, packets in these classes can be dropped by using Random Early packets. When there are no $\mathrm{EF}$ packets left, packets from AF classes can be sent. The AF class packets to be picked from the class is performed by using WFQ mechanism. The weights properly chosen reflect the guaranteed
AF Classes of queues of $1,2,3$, and 4 , with three different loss levels at each queue

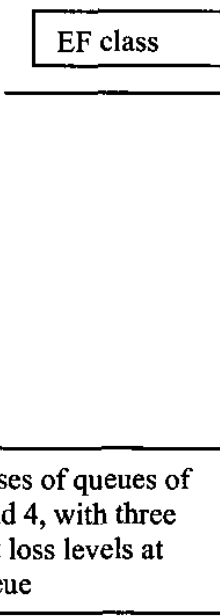

BE class
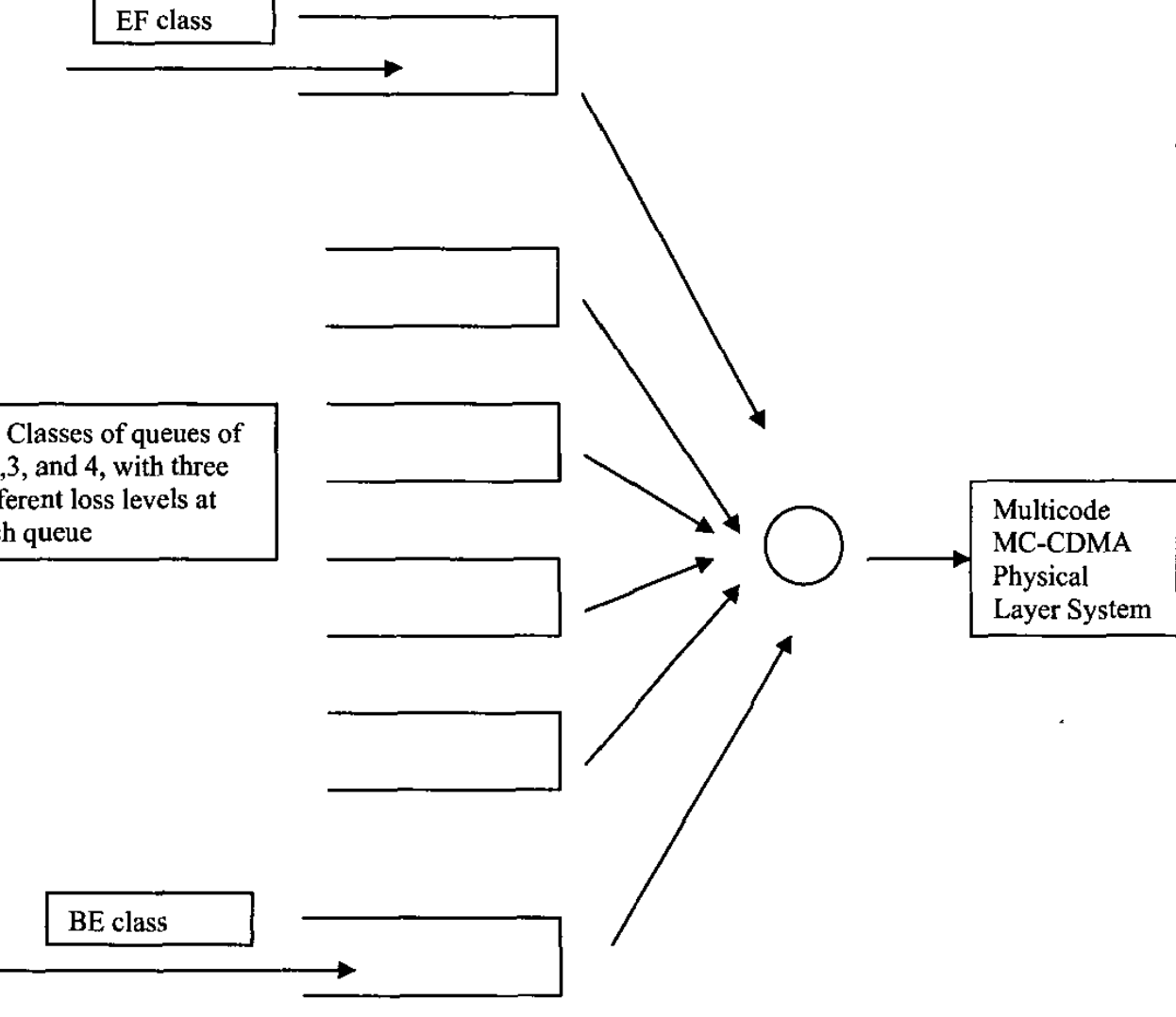

Figure 1: A Diffserv Queue Architecture with packets delivered to Wireless Link

Detection(RED) algorithm. A typical architecture of IP Diffserv QoS classes can be as shown. Note that in the following figure we are showing only one mobile terminal queue and MAC structure. Similar queue distribution exists at each mobile station and they all send data to the receiver structure of base station.

As shown in Figure 1, the server which sends packets to wireless multicode MC-CDMA transmitting system, gives priority to EF class throughput to be supported for each $\mathrm{AF}$ queue.We use RED mechanism for selectively dropping the packet from the AF queues. Note that the packet loss occurring in the queue is due to buffer overflow. But packet loss can also occur, when the errors on the bit cannot be corrected. To prevent such losses, the base station when cannot have error free packet can send a NACK to mobile station. The mobile station can resend the packet depending on the type of packet. If the packet is a real time packet 
and if the maximum allowable delay is already exceeded, such packets can be dropped. But, for non-real-time packet which has to meet throughput requirement(AF class of packets) and certain loss criterion, and $\mathrm{BE}$ packet which can tolerate delay, can be retransmitted at the Physical layer.

In order to ensure the throughput requirement of AF classes and timely delivery of EF classes, the power control and rate control can be performed. A typical power control and rate control mechanism strives hard to deliver the packets existing at the physical buffer of the mobile stations. Depending on the nature of packets, the mobile stations can send the SINR request to the base station. By assigning appropriate power to each mobile station in addition to assigning multiple codes, the base station can exercise rate control by choosing appropriate modulation scheme, such as, QPSK, 16QAM, 64QAM, etc. The ARM function chooses rate control adaptively using both multiple codes and modulation schemes depending on the requests from mobile stations which indicates SINR and priority of the traffic. At times, when the traffic configuration changes, ARM chooses appropriate weighting factors for WFQ mechanism of AF queues and updates the priority parameters appropriately.

We use OPNET package, to simulate the system and based on heuristic functions chosen for ARM, exercise different controls in order to maximize system capacity and to meet the required QoS at Diffserv level. We define throughput requirement for each $\mathrm{AF}$ queues and probability of loss for three different levels at each queue, in our simulation. We also define the delay requirement of real-time traffic of $\mathrm{EF}$ class. Based on these parameters, the simulation uses a set of heuristic functions, to choose priority level for packets to be transmitted at each mobile station and SINR in order to meet required BER.

\section{CONCLUSION}

Our initial study shows that we can achieve very good utilization of the wireless capacity when the different parameters of interest can be adjusted appropriately. Since there are so many parameters, coming out with particular rules by analytical means can become formidable task. Our future work will include deriving such rules based on simplistic assumptions. As, this field is an emerging area for providing high speed wireless data communication of the order of Gbps, our insights can result in the recommendations for the yet to be decided standards of next generation wireless networks.

\section{References}

[1] R. Berezdevin, R. Breinig, and R. T. Raytheon, "Next Generation Wireless Communication Concepts and Technologies", IEEE Communications Magazine, March 2002, pp. 108116.

[2] R. Kimura and F. Adachi, "Comparison of OFDM and multi-code MC-CDMA in Frequency Selective Fading Channel", Electronics Letters, $6^{\text {th }}$ February 2003, Vol. 39, No. 3.

[3] S.Hara, and R. Prasad, "Overview of Multicarrier CDMA", IEEE Communications Magazine, December 1997, pp. 126-133.

[4] E. A. Sourour, and M. Nakagawa, "Performance of Orthogonal Multicarrier CDMA in a Multipath Fading Channel', IEEE Transactions on Communications, Vol. 44, No. 3, March 1996, pp. 356-367.

[5] M. Elaoud and P. Ramanathan, "Adaptive Allocation of CDMA Resources for Network Level QoS Assurances", Mobicom 2000, pp. 191-199.

[6] A. Harada, S. Abeta, and M. Sawahashi, "Adaptive Radio Parameter Control Considering QoS for Forward Link OFCDM Wireless Access", IEEE Vehicular Technology Conference, Birmingham, Al, May 6-9, 2002, pp. 1175-1179. 